\title{
ENERGY SPLITTING*
}

\author{
BY \\ DAVID G. COSTA (Universidade de Brasilia) \\ AND
}

WALTER A. STRAUSS (Brown University)

1. Introduction. In Huygens' theory of light, waves spread equally in all directions until they are reflected or refracted. Huygens wrote: "Dans une grande distance du corps lumineux une infinité d'ondes, quoique issuës de points differents de ce corps, s'unissent en sorte que sensiblement elles ne composent qu'une onde seule ..." [11]. Thus a solution of the wave equation $\varphi_{t t}=c^{2} \Delta \varphi$ which is initially localized should look like a single expanding spherical wave after the elapse of a sufficiently long time.

In this article we express Huygens' idea in terms of the asymptotic behavior of various parts of the energy $\mathscr{E}$ of the wave. Namely,

$$
\int\left|c \nabla \varphi+\frac{x}{r} \varphi_{t}\right|^{2} d x \rightarrow 0 \text { as } t \rightarrow+\infty
$$

for all solutions with finite energy, the integration being taken over all space. Thus the spatial gradient $\nabla \varphi$ points approximately away from (or towards) the origin, just as for a spherical wave. Since $\mathscr{E}$ is a constant, it follows from (1) that all the energy is carried by its forward component $\mathscr{E}_{f}$ :

$$
\mathscr{E}_{f}=\frac{1}{4} \int\left|c \varphi_{r}-\varphi_{t}\right|^{2} d x \rightarrow \mathscr{E}=\frac{1}{2} \int\left[c^{2}|\nabla \varphi|^{2}+\left|\varphi_{t}\right|^{2}\right] d x
$$

as $t \rightarrow+\infty$. We call this phenomenon energy splitting. It can be interpreted as stating that asymptotically all the energy propagates along the light rays issuing from the origin. This is an interpretation of the duality between waves and rays. It also follows from (1) that the kinetic and potential energies are asymptotically equal, a fact first observed by Lax and Phillips [13] and Brodsky [3] and widely generalized since then [4].

For Maxwell's equations, the same ideas lead to the conclusion that $E \sim-(x / r) \times H$ and $H \sim(x / r) \times E$ as $t \rightarrow+\infty$. It follows that the vectors $E, H$ and $x$ are asymptotically orthogonal. Furthermore, the electric and magnetic fields are equi-partitioned, a fact first specifically observed by Dassios [6]. Thus any electromagnetic field looks asymptotically like a pure radiation field.

The wave equation and Maxwell's equations are discussed in Sec. 3 of this article. In Sec. 4 we discuss some other examples, including the equations for isotropic elastic waves, Dirac's equations, the wave equation and Maxwell's equations in the presence of a

* Received May 9, 1980. The first author was supported by Conselho Nacional de Desenvolvimento Científico e Tecnológico ( $\mathrm{CNPq})$ and the second author by National Science Foundation Grant MCS 79-01965. 
reflecting obstacle, nonlinear wave equations and the Yang-Mills equations. It was in the study of the Yang-Mills equations that we first noticed the phenomenon of energy splitting [10]. The method used in [10] depends on the conformal invariance of the equations.

In Sec. 2 we derive the energy splitting for an isotropic symmetric hyperbolic system

$$
\frac{\partial u}{\partial t}+\sum_{k=1}^{n} A_{k} \frac{\partial u}{\partial x_{k}}=0 .
$$

We use a method which does not require conformal invariance but does require the system to be linear. We use the explicit representation of the solutions of (2) via the Radon transform. The energy splitting is then expressed in the following manner. If $u$ is an $L^{2}$ solution and $u^{(j)}$ is the part of $u$ which travels at a characteristic speed $\lambda_{j} \neq 0$, then

$$
\int\left|\lambda_{j} u^{(j)}-\sum_{k=1}^{n} \frac{x_{k}}{r} A_{k} u^{(j)}\right|^{2} d x \rightarrow 0
$$

as $t \rightarrow+\infty$.

Energy splitting is closely related to the well-known statement that "every solution looks asymptotically like a pure radiation field." For the wave equation in three dimensions, this means that

$$
\varphi(x, t) \sim \frac{1}{r} f\left(c t-r, \frac{x}{r}\right) \text { as } t \rightarrow+\infty
$$

where $f$ is called the radiation field or the asymptotic wave profile of $\varphi$. This was first proved in a pointwise sense by Friedlander [9] for arbitrary smooth solutions. In the energy sense, (3) was proved by Lax and Phillips (see [14], p. 179). They proved that the total energy of the difference between the two sides in (3) goes to zero as $t \rightarrow \infty$. Once it is known that (3) can be differentiated, the energy splitting follows. Thus (1) is easily obtainable from known facts in several ways. However, it apparently has never been explicitly observed before.

After this paper was completed we learned that the work of Wilcox [21] makes a far-reaching generalization of (3) to symmetric hyperbolic systems with bounded wave speeds. We are grateful to the person who pointed this out to us. Wilcox uses the Fourier, rather than the Radon, transform. It follows that the energy splitting is valid for such systems.

We have also received the work of Strichartz [19] which was motivated by our announcements $[5,18]$. He presents some closely related results from a Fourier-analytical point of view.

We thank R. Glassey for many useful discussions and P. Letelier for conversations on the physical aspects of the problem. Part of this work was carried out while the first author was visiting the Mathematics Department at Brown University, for whose hospitality he is grateful.

2. Main theorem. Consider the isotropic symmetric hyperbolic system (2), where $x=\left(x_{1}, \ldots, x_{n}\right) \in \mathbb{R}^{n}$, each $A_{k}$ is an $N \times N$ hermitian matrix and the characteristic speeds $\lambda_{j}$ are constant (see the Appendix for our terminology). Let

$$
A(\omega)=\sum_{k=1}^{n} A_{k} \omega_{k} \quad \text { for } \omega=\left(\omega_{1}, \ldots, \omega_{n}\right) \in \mathbb{R}^{n}, \quad|\omega|=1 .
$$


The characteristic speeds are the eigenvalues of $A(\omega)$. In what follows we shall denote various constants by the same letter $c$.

TheOREM. Let $u=u(x, t)$ be any $L^{2}$ solution of (2). Let $\lambda_{j}$ be a positive characteristic speed. Let $u^{(j)}=u^{(j)}(x, t)$ be the part of $u$ which travels at speed $\lambda_{j}$. Then

$$
\left\lfloor\lambda_{j} I \mp A\left(\frac{x}{r}\right)\right\rfloor u^{(j)} \rightarrow 0 \quad \text { in } \quad L^{2}\left(\mathbb{R}^{n}\right)^{v} \quad \text { as } t \rightarrow \pm \infty .
$$

Proof. We may restrict ourselves to the case $t \rightarrow+\infty$. We have the formula (see the Appendix)

$$
u^{(j)}(x, t)=c \int P_{j}(\omega) \partial_{s}^{n-1} H \hat{f}\left(x \cdot \omega-\lambda_{j} t, \omega\right) d \omega
$$

where $H$ is the Hilbert transform if $n$ is even and $H$ is the identity if $n$ is odd. Let $v=\lambda_{j} u^{(j)}-A(x / r) u^{(j)}$. Because $A(\omega) P_{j}(\omega)=\lambda_{j} P_{j}(\omega)$, we have

$$
v(x, t)=c \int\left\lfloor A(\omega)-A\left(\frac{x}{r}\right)\right\rfloor P_{j}(\omega) \partial_{s}^{n-1} H \hat{f}\left(x \cdot \omega-\lambda_{j} t, \omega\right) d \omega .
$$

We wish to prove that $\|v(t)\| \rightarrow 0$ as $t \rightarrow+\infty$, where $\|\cdot\|$ denotes the norm in $L^{2}\left(\mathbb{R}^{n}\right)^{N}$. Since

$$
c\|v(t)\| \leq\left\|u^{(j)}(t)\right\| \leq\|f\|,
$$

it is enough to prove it for $f$ in a dense set of $L^{2}\left(\mathbb{R}^{n}\right)^{x}=\mathscr{L}$.

In case $n$ is odd, we use the fact that the map $f \mapsto \alpha_{n} \partial_{s}^{(n-1) / 2} \hat{f}, \alpha_{n}=2^{-1 / 2}(2 \pi)^{(1-n) / 2}$, takes $\mathscr{L}$ unitarily onto the space $\mathscr{K}$ of even (or odd, depending on the parity of $(n-1) / 2)$ functions in $L^{2}\left(\mathbb{R} \times S^{n-1}\right)$. So we may take $\partial_{s}^{(n-1) / 2} \hat{f}$ in a set with dense linear span in $\mathscr{K}$. We choose

$$
\partial_{s}^{(n-1) / 2} \hat{f}(s, \omega)=\varphi^{\prime}(s) \psi(\omega)
$$

where $\psi \in C^{\infty}\left(S^{n-1}\right)^{N}$ and $\varphi \in C_{0}^{\infty}(\mathbb{R})$ and $\varphi^{\prime}=d \varphi / d s$, noting that $\left\{\varphi^{\prime} \mid \varphi \in C_{0}^{\infty}(\mathbb{R})\right\}$ is dense in $L^{2}(\mathbb{R})$. Putting (5) into (4), we get

$$
v(x, t)=\int \eta(\omega) \partial_{s}^{m} \varphi\left(x \cdot \omega-\lambda_{j} t\right) d \omega
$$

where $\eta(\omega)=c[A(\omega)-A(x / r)] P_{j}(\omega) \psi(\omega)$ and $m=(n+1) / 2$.

In case $n$ is even, we will also show that $v$ may be taken in the form (6), but now with $m=n / 2$. Indeed, the map $f \mapsto \alpha_{n} H^{n / 2}\left|\partial_{s}\right|^{(n-1) / 2} \hat{f}$ takes $\mathscr{L}$ unitarily onto the space $\mathscr{K}$ of even (or odd) functions in $L^{2}\left(\mathbb{R} \times S^{n-1}\right)$ (see the Appendix). So we may take $H^{n / 2}\left|\partial_{s}\right|^{(n-1) / 2} \hat{f}$ in a set with dense linear span in $\mathscr{K}$. We choose

$$
H^{n / 2}\left|\partial_{s}\right|^{(n-1) / 2} \hat{f}(s, \omega)=\left|\partial_{s}\right|^{1 / 2} \varphi(s) \psi(\omega)
$$

where $\varphi$ and $\psi$ are as above. It is easily seen by Fourier transform that, since $C_{0}^{\infty}(\mathbb{R})$ is dense in $L^{2}(\mathbb{R}),\left\{\left|\partial_{s}\right|^{1 / 2} \varphi \mid \varphi \in C_{0}^{\infty}(\mathbb{R})\right\}$ is also dense. Now (7) implies that

$$
\partial_{s}^{n-1} H \hat{f}=\partial_{s}^{n / 2} H \partial_{s}^{(n-2) / 2} \hat{f}=\partial_{s}^{m}\left|\partial_{s}\right|^{-1 / 2} H^{n / 2}\left|\partial_{s}\right|^{(n-1) / 2} \hat{f}=\partial_{s}^{m} \varphi(s) \psi(\omega)
$$

where $m=n / 2$. Thus (4) and (7) imply (6). This argument is essentially taken from [1].

We now have Eq. (6). We perform the integration in (6) over the variables orthogonal to $x$ and write $\lambda_{j}=\lambda$. Thus 


$$
v(x, t)=c \int_{-1}^{1} \bar{\eta}(s) \partial^{m} \varphi(s r-\lambda t)\left(1-s^{2}\right)^{(n-3) / 2} d s
$$

where $\bar{\eta}(s)$ is the average of $\eta(\omega)$ over the "zone" $\{\omega \mid \omega \cdot x / r=s\}$ (see page 8 of [12], for instance). We may write this formula as

$$
v(x, t)=\int_{-1}^{1} \chi(s) \partial^{m} \varphi(s r-\lambda t) d s,
$$

or yet,

$$
v(x, t)=v^{-}(x, t)+v^{+}(x, t),
$$

where $v^{-}$and $v^{+}$correspond to integrating over $-1 \leq s \leq 0$ and $0 \leq s \leq 1$, respectively. Recalling that $\eta(x / r)=0$, we have $\bar{\eta}(1)=0$. Hence

$$
\partial^{j} \chi(s)=0\left((1-s)^{(n-1) / 2-j}\right) \text { as } s \uparrow 1,
$$

for $j \geq 0$. Also $\chi \in C^{\infty}((-1,1))^{v}$ and $\varphi \in C_{0}^{\infty}(\mathbb{R})$.

Let $\varphi$ vanish outside the interval $[-R, R]$. Let $t>R / \lambda$. Then $v^{-}=0$ because, for $-1 \leq s \leq 0, s r-\lambda t \leq-\lambda t<-R$ so that $(s r-\lambda t)$ is outside the support of $\varphi$. Thus

$$
v(x, t)=v^{+}(x, t)=\int_{0}^{1} \chi(s) \partial^{m} \varphi(s r-\lambda t) d s .
$$

We integrate by parts $m$ times. The resulting integrated terms are

$$
v_{1}^{+}=-\left.\sum_{j=0}^{m-1}(-r)^{-j-1} \partial_{s}^{m-j-1} \varphi(s r-\lambda t) \partial^{j} \chi(s)\right|_{0} ^{1}
$$

with the terms at $s=0$ vanishing by the same reason as above. And, because of (9), the part of $v_{1}^{+}$at $s=1$ vanishes so long as $j<(n-1) / 2$. Therefore $v_{1}^{+}=0$ if $n$ is even and

$$
v_{1}^{+}=-(-r)^{-m} \varphi(r-\lambda t) \partial^{(n-1) / 2} \chi(1)
$$

if $n$ is odd.

After the integrations by parts, the remaining integral is

$$
v_{2}^{+}=(-r)^{-m} \int_{0}^{1} \varphi(s r-\lambda t) \partial^{m} \chi(s) d s .
$$

We observe that

$$
v=v^{+}=v_{1}^{+}=v_{2}^{+}=0 \text { for } r-\lambda t<-R
$$

because $\varphi$ vanishes on the interval $(-\infty,-R)$. For $n$ odd, we have

$$
|v|=\left|v^{+}\right| \leq\left|v_{1}^{+}\right|+\left|v_{2}^{+}\right| \leq c r^{-m}=c r^{-(n+1) / 2} .
$$

For $n$ even, we have

$$
|v|=\left|v^{+}\right|=\left|v_{2}^{+}\right| \leq c r^{-n / 2} \int_{0}^{1}|\varphi(s r-\lambda t)|(1-s)^{-1 / 2} d s
$$

by (9). Let $w$ be this integral. We claim that $|w| \leq c r^{-1 / 2}$. Indeed, if $r>\lambda t+R$, we introduce $\sigma=s r-\lambda t$ to get 


$$
|w| \leq c r^{-1 / 2} \sup |\varphi| \int_{-R}^{R}(R-\sigma)^{-1 / 2} d \sigma \leq c r^{-1 / 2}
$$

If $r \leq \lambda t+R$, we estimate

$$
|w|=\int_{1-2 R / r}^{1}|\varphi(s r-\lambda t)|(1-s)^{-1 / 2} d s \leq \sup |\varphi| \int_{0}^{2 R / r} \tau^{-1 / 2} d \tau=c r^{-1 / 2}
$$

Therefore we have

$$
|v(x, t)| \leq c r^{-(n+1) / 2}
$$

in every case. By (10) and (11) we obtain

$$
\|v(t)\|^{2}=\int|v(x, t)|^{2} d x \leq c \int_{r>\lambda t-R} r^{-n-1} d x=c \int_{\lambda t-R}^{\infty} r^{-2} d r=O\left(t^{-1}\right) \text { as } \quad t \rightarrow+\infty .
$$

This completes the proof.

\section{The wave and Maxwell's equations.}

A. The wave equation.

$$
\varphi_{t t}-c^{2} \Delta \varphi=0 \quad\left(x \in \mathbb{R}^{n}\right) .
$$

This takes the form (2) of a symmetric hyperbolic system if we let $N=n+1, u=\left(u_{0}, u_{1}\right.$, $\left.\ldots, u_{n}\right)$,

$$
u_{0}=\partial_{t} \varphi=\varphi_{t}, u_{i}=c \partial_{i} \varphi \quad(i=1, \ldots, n),
$$

$A_{k}=$ the matrix with entry $(-c)$ in the $o k$ and $k o$ positions and zeros elsewhere. Then $A(\omega)=$ the matrix with entry $\left(-c \omega_{i}\right)$ in the oi and io positions $(i=1, \ldots, n)$ and zeros elsewhere. Thus the characteristic speeds (eigenvalues of $A(\omega))$ are $+c,-c, 0, \ldots, 0$. And each solution has the representation $u=u^{(0)}+u^{(1)}$ where $u^{(0)}$ travels at speed zero and $u^{(1)}$ travels at speed $\pm c$. Because of $(12), u^{(0)}=0$. Thus $u=u^{(1)}$. The theorem states that

$$
c u-A\left(\frac{x}{r}\right) u \rightarrow 0 \quad \text { in } \quad L^{2}\left(\mathbb{R}^{n}\right)^{n+1} \quad \text { as } \quad t \rightarrow+\infty .
$$

Now

$$
\begin{gathered}
A\left(\frac{x}{r}\right) u=\left(\begin{array}{cc}
0 & -c(x / r) \\
-c(x / r) & 0
\end{array}\right)\left(\begin{array}{c}
\varphi_{t} \\
c \nabla \varphi
\end{array}\right)=\left(\begin{array}{c}
-c^{2} \varphi_{r} \\
-c(x / r) \varphi_{t}
\end{array}\right), \\
u-\frac{1}{c} A\left(\frac{x}{r}\right) u=\left(\begin{array}{c}
\varphi_{t}+c \varphi_{r} \\
c \nabla \varphi+(x / r) \varphi_{t}
\end{array}\right) .
\end{gathered}
$$

Therefore our theorem takes the form

$$
\sum_{i=1}^{n} \int\left|c \partial_{i} \varphi+\frac{x_{i}}{r} \varphi_{t}\right|^{2} d x \rightarrow 0
$$

Another way to think of (13) is to split up the energy

$$
\mathscr{E}=\frac{1}{2} \int\left(\varphi_{t}^{2}+c^{2}|\nabla \varphi|^{2}\right) d x
$$


as

$$
\mathscr{E}=\mathscr{E}_{f}+\mathscr{E}_{b}+\mathscr{E}_{a}
$$

where

$$
\begin{aligned}
& \mathscr{E}_{f}=\frac{1}{4} \int\left(\varphi_{t}-c \varphi_{r}\right)^{2} d x=\text { forward part } \\
& \mathscr{\delta}_{b}=\frac{1}{4} \int\left(\varphi_{t}+c \varphi_{r}\right)^{2} d x=\text { backward part } \\
& \mathscr{E}_{a}=\frac{1}{2} \int c^{2}\left(|\nabla \varphi|^{2}-\varphi_{r}^{2}\right) d x=\text { angular part. }
\end{aligned}
$$

Then (13) is equivalent to the statement that

$$
\mathscr{E}_{b}+\mathscr{E}_{a} \rightarrow 0 \text { as } t \rightarrow+\infty .
$$

Thus $\mathscr{E}_{f}$ carries all the energy as $t \rightarrow+\infty$.

In particular, we get equipartition of energy:

$$
\int\left(c^{2}|\nabla \varphi|^{2}-\varphi_{t}^{2}\right) d x=\sum_{i} \int\left(c^{2}\left|\partial_{i} \varphi\right|^{2}-\left|\frac{x_{i}}{r} \varphi_{t}\right|^{2}\right) d x \rightarrow 0
$$

hence

$$
\frac{c^{2}}{2} \int|\nabla \varphi|^{2} d x \rightarrow \frac{1}{2} \mathscr{E} \quad \text { and } \quad \frac{1}{2} \int \varphi_{t}^{2} d x \rightarrow \frac{1}{2} \mathscr{E}
$$

Also

$$
c \int \varphi_{t} \varphi_{r} d x \rightarrow-\mathscr{E} .
$$

Equipartition of the energy was proved first (to our knowledge) by Lax and Phillips [13].

We now present the conformal method for proving energy splitting (13). The wave equation is invariant under the conformal transformations. This leads to various conservation laws. One of them (coming from invariance under inversion and time translation) is

$$
\left.\int \mid\left(t^{2}+r^{2}\right) \frac{1}{2}\left(\varphi_{t}^{2}+|\nabla \varphi|^{2}\right)+2 \operatorname{tr} \varphi_{t} \varphi_{r}+(n-1) t \varphi_{t} \varphi-\frac{n-1}{2} \varphi^{2}\right\rfloor d x=\text { constant }
$$

This identity, due to Morawetz (see Appendix in [13]), can be proved directly by multiplying the wave equation by $\left(t^{2}+r^{2}\right) \varphi_{t}+2 \operatorname{tr} \varphi_{r}+(n-1) t \varphi$ and integrating over space. It is valid so long as $r \varphi_{t}$ and $r \nabla \varphi$ are square-integrable at fixed times. It can be rewritten as

$$
\begin{aligned}
& \frac{1}{2} \int\left(t^{2}+r^{2}\right)\left(c^{2}|\nabla \varphi|^{2}-\varphi_{r}^{2}\right) d x+\frac{1}{4} \int(t+r)^{2}\left(\varphi_{t}+c \varphi_{r}+c \frac{n-1}{2 r} \varphi\right)^{2} d x \\
& +\frac{1}{4} \int(t-r)^{2}\left(\varphi_{t}-c \varphi_{r}-c \frac{n-1}{2 r} \varphi\right)^{2} d x+\frac{(n-1)(n-3)}{4} \int\left(c^{2} t^{2}+r^{2}\right) \frac{\varphi^{2}}{r^{2}} d x=\text { constant. }
\end{aligned}
$$


However, at least for a dense class of solutions, $\int \varphi^{2} d x$ is bounded and $\int \varphi^{2} / r^{2} d x=$ $O\left(t^{-2}\right)$ as $t \rightarrow+\infty$. Hence

$$
\int\left(|\nabla \phi|^{2}-\varphi_{r}^{2}\right) d x+\int\left(\varphi_{t}+c \varphi_{r}\right)^{2} d x=O\left(t^{-2}\right)
$$

as $t \rightarrow+\infty$.

B. Maxwell's equations.

$$
\begin{aligned}
& \partial_{t} E-\nabla \times H=0 \\
& \partial_{t} H+\nabla \times E=0
\end{aligned} \quad\left(x \in \mathbb{R}^{3}\right) .
$$

It takes the form (2) if we let $N=6$, the first three components of $u$ be the components of $E$ and the last three components of $u$ be the components of $H$. Then

$$
\begin{gathered}
\sum_{k=1}^{3} A_{k} \partial_{k}=\left(\begin{array}{cc}
0 & - \text { curl } \\
+\operatorname{curl} & 0
\end{array}\right), \\
A(\omega)=\left(\begin{array}{cc}
0 & B(\omega) \\
B(\omega) & 0
\end{array}\right), \quad B(\omega)=\left(\begin{array}{ccc}
0 & \omega_{3} & -\omega_{2} \\
-\omega_{3} & 0 & \omega_{1} \\
\omega_{2} & -\omega_{1} & 0
\end{array}\right)=-\omega \times .
\end{gathered}
$$

The eigenvalues of $A(\omega)$ are $1,-1$ and zero. Every solution of (17) has the form $u^{(0)}+u^{(1)}$. Then $u=u^{(1)}$ if and only if

$$
\nabla \cdot E=\nabla \cdot H=0
$$

If (18) holds initially, it holds at all times. From now on we only consider solutions of (17), (18). Now

$$
u-A\left(\frac{x}{r}\right) u=\left(\begin{array}{l}
E+(x / r) \times H \\
H-(x / r) \times E
\end{array}\right) \rightarrow 0 \quad \text { in } \quad L^{2}\left(\mathbb{R}^{3}\right)^{6} .
$$

Thus

$$
\int|| E+\frac{x}{r} \times\left. H\right|^{2}+\left|H-\frac{x}{r} \times E\right|^{2} \mid d x \rightarrow 0
$$

as $t \rightarrow+\infty$. It follows that the three vectors $E, H$ and $x / r$ become mutually orthogonal in the sense that

$$
\int\left[\left(\frac{x}{r} \cdot E\right)^{2}+\left(\frac{x}{r} \cdot H\right)^{2}+E \cdot H\right] d x \rightarrow 0
$$

as $t \rightarrow+\infty$. This is found by taking the dot product of $E+(x / r) \times H$ with $x / r$ and $H-(x / r) \times E$ with $x / r$ and $E$. On the other hand, if we dot $E+(x / r) \times H$ with $E$, $H-(x / r) \times E$ with $H$, and subtract, we see that

$$
\int\left(|E|^{2}-|H|^{2}\right) d x \rightarrow 0 \text {. }
$$

This is equipartition of energy between the electric and magnetic fields: 


$$
\frac{1}{2} \int|E|^{2} d x \rightarrow \frac{1}{2} \mathscr{E}, \quad \frac{1}{2}\left\lceil|H|^{2} d x \rightarrow \frac{1}{2} \mathscr{E}\right.
$$

where $\mathscr{E}=\frac{1}{2} \int\left(|E|^{2}+|H|^{2}\right) d x=$ constant. We can also split up the energy as (14) with

$$
\begin{aligned}
& \mathscr{E}_{f}=\frac{1}{4} \int\left(|E \alpha-H \beta|^{2}+|E \beta+H \alpha|^{2}\right) d x, \\
& \mathscr{E}_{b}=\frac{1}{4} \int\left(|E \alpha+H \beta|^{2}+|E \beta-H \alpha|^{2}\right) d x, \\
& \mathscr{E}_{a}=\frac{1}{2} \int\left(|E \omega|^{2}+|H \omega|^{2}\right) d x,
\end{aligned}
$$

where $\omega=x / r$ and $\omega, \alpha, \beta$ form an orthonormal set of vectors in $\mathbb{R}^{3}$ with $\omega=\alpha \times \beta$. Then (19) is equivalent to the statement that $\mathscr{E}_{b}+\mathscr{E}_{a} \rightarrow 0$ as $t \rightarrow+\infty$.

The conformal invariance of Maxwell's equations (17), (18) leads to the identity [10]

$$
\int\left[\left(t^{2}+r^{2}\right) \frac{1}{2}\left(|E|^{2}+|H|^{2}\right)+2 t x \cdot H \times E\right] d x=\text { constant } .
$$

This identity is valid for solutions such that $r E$ and $r H$ are square-integrable at fixed times. It can be rewritten as

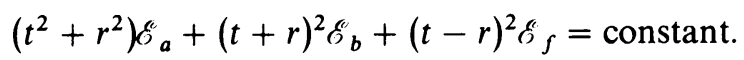

Therefore

$$
\mathscr{E}_{a}+\mathscr{E}_{b}=O\left(t^{-2}\right) \quad \text { as } t \rightarrow+\infty, \quad \mathscr{E}_{a}+\mathscr{E}_{f}=O\left(t^{-2}\right) \text { as } t \rightarrow-\infty
$$

This was the first proof of the energy splitting [10]. It also implies that, for $\varepsilon>0$,

$$
\int_{r<(1-\varepsilon) t}\left(|H|^{2}+|E|^{2}\right) d x=O\left(t^{-2}\right)
$$

which means that all the energy is radiating at the speed of light (cf. [2]).

\section{Other applications.}

C. Isotropic elastic waves. We have

$$
\partial_{t}^{2} u-\mu \Delta u-(\lambda+\mu) \nabla(\nabla \cdot u)=0
$$

where $x \in \mathbb{R}^{3}, u$ is a 3-vector field, and $\lambda$ and $\mu$ are positive constants. We decompose $u$ as the sum of a shear wave and a pressure wave:

$$
u=\nabla \times w+\nabla v=\operatorname{curl} w+\operatorname{grad} v
$$

where $w$ is a vector field and $v$ a scalar field. Substituting (22) into (21), we obtain

$$
\left(\partial_{t}^{2}-\mu \Delta\right)(\nabla \times w)+\left(\partial_{t}^{2}-(\lambda+2 \mu)\right)(\nabla v)=0 .
$$

Thus the shear wave $\nabla \times w$ propagates at speed $\mu$ and the pressure wave $\nabla v$ propagates at speed $(\lambda+2 \mu)$. The two components in (22) are orthogonal to each other. Therefore our result on energy splitting can be stated as: 


$$
\sum_{i=1}^{3} !\left|\left(\frac{x_{i}}{r} \partial_{t}+\mu \partial_{i}\right)(\nabla \times w)\right|^{2} d x \rightarrow 0, \quad \sum_{i=1}^{3} !\left|\left(\frac{x_{i}}{r} \partial_{t}+(\lambda+2 \mu) \partial_{i}\right)(\nabla v)\right|^{2} d x \rightarrow 0
$$

as $t \rightarrow+\infty$. Equipartition of the energy in finite time is proved for this case by Dassios [7].

D. Dirac's equation with zero mass. We write it in the explicit representation

$$
\frac{\partial u}{\partial t}=\sum_{j=1}^{3} A_{j} \frac{\partial u}{\partial x_{j}}, \quad A_{j}=\left(\begin{array}{cc}
0 & \sigma_{j} \\
\sigma_{j} & 0
\end{array}\right)
$$

where $N=4$ and each $A_{j}$ is written in $2 \times 2$ blocks with

$$
\sigma_{1}=\left(\begin{array}{ll}
0 & 1 \\
1 & 0
\end{array}\right), \quad \sigma_{2}=\left(\begin{array}{rr}
0 & i \\
-i & 0
\end{array}\right), \quad \sigma_{3}=\left(\begin{array}{rr}
1 & 0 \\
0 & -1
\end{array}\right)
$$

The characteristic speeds are +1 and -1 , each being a double root. Therefore, $u=u^{(1)}$ and

$$
u-\left(\sum_{j=1}^{3} \frac{x_{j}}{r} A_{j}\right) u \rightarrow 0 \quad \text { in } \quad L^{2}\left(\mathbb{R}^{3}\right)^{4}
$$

Let $x_{j} / r=\omega_{j}(j=1,2,3)$ and

$$
\sigma=\sum_{j=1}^{3} \omega_{j} \sigma_{j}=\left(\begin{array}{cc}
\omega_{3} & \omega_{1}+i \omega_{2} \\
\omega_{1}-i \omega_{2} & -\omega_{3}
\end{array}\right)
$$

If we write

$$
v=\left(\begin{array}{l}
u_{1} \\
u_{2}
\end{array}\right) \quad \text { and } \quad w=\left(\begin{array}{l}
u_{3} \\
u_{4}
\end{array}\right)
$$

then (23) takes the form

$$
\int|v-\sigma \omega|^{2} d x+\int|w-\sigma v|^{2} d x \rightarrow 0 \text { as } t \rightarrow+\infty .
$$

Now we $\operatorname{dot} v-\sigma w$ with $v$ and $w-\sigma v$ with $\omega$, and subtract to obtain

$$
\int\left(|v|^{2}-|w|^{2}\right) d x=\int\left(\left|u_{1}\right|^{2}+\left|u_{2}\right|^{2}-\left|u_{3}\right|^{2}-\left|u_{4}\right|^{2}\right) d x \rightarrow 0 .
$$

In Dirac's notation, $\psi=u$, this means that $\int \psi \psi d x \rightarrow 0$. This is equipartition of energy.

E. Exterior domains. We begin with a remark. Suppose we consider a perturbation of the system (2). Suppose we can prove that, for all solutions $v(t)$ of the perturbed system, there exists a solution $u(t)$ of the original system (2) such that

$$
v(t)-u(t) \rightarrow 0 \text { in } \quad L^{2}\left(\mathbb{R}^{n}\right)^{v} \text { as } t \rightarrow+\infty .
$$

Then the result on energy splitting is also valid for the perturbed system. The hypothesis just made is a standard situation in scattering theory.

Now consider the wave equation in the exterior $\Omega$ of a bounded obstacle:

$$
\left(\partial_{t}^{2}-\Delta\right) \varphi=0, \quad x \in \Omega ; \quad u=0 \quad \text { or } \partial \Omega
$$


Then the above remark is applicable $[13,15,20]$. We conclude that $(13)$ and its consequences are valid for solutions of $(25)$ (the integration is taken over $\Omega$ ). The conformal method also works and implies (16) in case the obstacle is star-shaped. More generally the obstacle may be allowed to move: $x \in \Omega(t), u=0$ on $\partial \Omega(t)$. Under certain assumptions on the motion, it can again be shown that (24) holds [17] and therefore the energy splits as before.

We can also consider Maxwell's equations in the exterior $\Omega$ of a perfect conductor:

$$
\left\{\begin{array}{l}
\partial_{t} E-\nabla \times H=0 \\
\partial_{t} H+\nabla \times E=0, \quad x \in \Omega ; \quad E_{\text {tang. }}=H_{\text {normal }}=0 \quad \text { on } \quad \partial \Omega . \\
\nabla \cdot E=\nabla \cdot H=0
\end{array}\right.
$$

Again, it is known that (24) holds (see Appendix in [13]) and we may conclude that (19) and its consequences are valid for solutions of (26).

F. Nonlinear wave equations. Consider

$$
\varphi_{t t}-\Delta \varphi+|\varphi|^{p-1} \varphi=0, \quad x \in \mathbb{R}^{n}
$$

If $n=p=3$, for instance, the remark at the beginning of Example $\mathrm{E}$ is applicable, if we regard (27) as a perturbation of the ordinary wave equation. Condition (24) was proved in [16].

The conformal method is more direct and flexible in this case. The identity (15) is valid as an inequality (the integral is $\leq$ constant), provided $p \geq 1+4(n-1)^{-1}, n \geq 3$. See [16].

G. The Yang-Mills equations. These are a nonlinear perturbation of Maxwell's equations. As the gauge group we take any compact Lie group. The conformal method works perfectly [10]. As a consequence, energy splitting is valid: every statement made above for Maxwell's equations is also valid for the Yang-Mills equations (only some care has to be taken about notation).

Appendix. The Radon transform is defined as follows. Let $f(x)$ be a function on $\mathbb{R}^{n}$. Let $s$ be a real number and $\omega$ a unit vector $\left((s, \omega) \in \mathbb{R} \times S^{n-1}\right)$. Then $\hat{f}(s, \omega)$ is defined as the integral of $f(x)$ over the hyperplane $\{x \mid x \cdot \omega=s\}$. Thus $\hat{f}(s, \omega)$ is an even function of the pair $(s, \omega)$. The Parseval relation can be written as follows. Let $F$ denote the (onedimensional) Fourier transform with respect to the variable $s$. Denote by $\left|\partial_{s}\right|$ the operator $F^{-1}|\sigma| F$. Then the map

$$
f \mapsto \alpha_{n}\left|\partial_{s}\right|^{(n-1) / 2} \hat{f} \quad\left(\alpha_{n}=2^{-1 / 2}(2 \pi)^{(1-n) / 2}\right)
$$

takes $L^{2}\left(\mathbb{R}^{n}\right)$ unitarily onto the space of even functions in $L^{2}\left(\mathbb{R} \times S^{n-1}\right)$. For a proof, see [15]. The Hilbert transform is the map $F^{-1}(\operatorname{sign} \sigma) F$.

Now consider the system (2). The eigenvalues $\lambda_{j}$ of the Hermitian matrix $A(\omega)$ are assumed to be constant. Multiplicity is allowed. Let $P_{j}(\omega)$ be the projection of $\mathbb{C}^{N}$ onto the subspace of $\lambda_{j}$-eigenvectors. Since (2) is homogeneous of degree one, the characteristic polynomial is either even or odd in $(\lambda, \omega)$, and hence the negatives of eigenvalues are also eigenvalues. Each $P_{j}(\omega)$ is an analytic function of $\omega$.

We consider solutions of (2) with initial condition $u(x, 0)=f(x)$. If $f \in L^{2}\left(\mathbb{R}^{n}\right)^{v}$ then a unique solution $u(x, t)$ exists with $\|u(t)\|=\|f\|$ for all $t$, where $\|\cdot\|$ denotes the $L^{2}$ norm. If $f$ is smooth, the solution is given by the formula [1] 


$$
u(x, t)=C_{n} \sum_{j} \int P_{j}(\omega) \partial_{s}^{n-1} H \hat{f}\left(x \cdot \omega-\lambda_{j} t, \omega\right) d \omega,
$$

where the sum is over all the eigenvalues (indexed by $j$ ), the integral is taken over the unit sphere $S^{n-1}=\{\omega|| \omega \mid=1\}$ and $H=I$ for $n$ odd, $H=$ Hilbert transform for $n$ even $\left(\partial_{s}=\partial / \partial s\right)$. Because $\hat{f}$ is even, the terms in (28) with $+\lambda_{j}$ and $-\lambda_{j}$ are equal to each other, and therefore we may consider the sum to be taken only over the non-negative $\lambda_{j}$. With this convention, we may write

$$
u=\sum_{j} u^{(j)}
$$

where $u^{(j)}$ is the $j$ th term in $(28)$.

\section{REFERENCES}

[1] G. S. S. Avila and D. G. Costa, Asymptotic properties of general symmetric hyperbolic systems, J. Funct. Anal. (to appear)

[2] C. Bardos and D. G. Costa, Decay along nonbicharacteristic rays of solutions of the first-order hyperbolic systems, J. Math. Pures Appl. 53, 427-435 (1974)

[3] A. R. Brodsky, On the asymptotic behavior of solutions of the wave equation, Proc. A.M.S. 18, 207-8 (1967)

[4] D. G. Costa, On partition of energy for uniformly propagative systems, J. Math. Anal. App. 58, 56-62 (1977)

[5] D. G. Costa and W. A. Strauss, Energy asymptotics of hyperbolic systems, A.M.S. Notices 26, A-434 (1979)

[6] G. Dassios, Equipartition of energy for Maxwell's equations, Quart. Appl. Math. (to appear)

[7] G. Dassios. Equipartition of energy in elastic wave propagation, Mech. Res. Comm. 6, 45-50 (1979)

[8] G. Dassios and E. Galanis, Asymptotic equipartition of kinetic and strain energy for elastic waves in anisotropic media, Quart. Appl. Math. 38, 121-128 (1980)

[9] F. G. Friedlander, On the radiation field of pulse solutions of the wave equation II, Proc. Roy. Soc. London 279A, 386-394 (1964)

[10] R. Glassey and W. A. Strauss, Decay of classical Yang-Mills fields, Comm. Math. Phys. 65, 1-13 (1979). Also, Propagation of the energy of Yang-Mills fields, in: Bifurcation phenomena in mathematical physics (ed. D. Bessis and C. Bardos), Reidel Publ. Co. (to appear)

[11] C. Huygens, Traité de la Lumière, Paris, 1690

[12] F. John, Plane waves and spherical means applied to partial differential equations, Interscience, 1955

[13] P. D. Lax and R. S. Phillips, Scattering theory, Academic Press, 1967

[14] P. D. Lax and R. S. Phillips, Decaying modes for the wave equation, Comm. Pure App. Math. 22, 737-787 (1969)

[15] P. D. Lax and R. S. Phillips, Scattering theory for the acoustic equation in an even number of space dimensions, Indiana U. Math. J. 22, 101-134 (1972)

[16] W. A. Strauss, Decay and asymptotics for $\square u=F(u)$, J. Funct. Anal. 2, 409-457 (1968)

[17] W. A. Strauss, Existence of the scattering operator for moving obstacles, J. Funct. Anal. 31, 255-262 (1979)

[18] W. A. Strauss, Some energy components tend to zero globally, A.M.S. Notices 26, A-431 (1979)

[19] R. S. Strichartz, Asymptotic behavior of waves (to appear)

[20] C. H. Wilcox, Scattering theory for the d'Alembert equation in exterior domains, Lecture Notes in Math. No. 442, Springer, 1975

[21] C. H. Wilcox, Asymptotic wave functions and energy distributions in strongly propagative anisotropic media, J. Math. Pures Appl. 57, 275-321 (1978) 